\title{
Cancer Patients in the Emergency Department: A "Nightmare" that Might Become a Virtuous Clinical Pathway
}

\author{
JACOPO MARIA LEGRAMANTE ${ }^{1}$, STEFANIA PELLICORI ${ }^{1,2}$, ANDREA MAGRINI ${ }^{3}$, \\ TIZIANA FRITTELLI ${ }^{4}$, VINCENZO FORMICA ${ }^{2}$ and MARIO ROSELLI ${ }^{2}$ \\ ${ }^{1}$ Emergency Department, ${ }^{2}$ Medical Oncology Unit, ${ }^{3}$ Medical Directorate, and \\ ${ }^{4}$ General Directorate, Tor Vergata University Hospital, Rome, Italy
}

\begin{abstract}
Background/Aim: Emergency departments (EDs) often face overcrowding issues while simultaneously confronting with the increasing clinical needs of patients, such as cancer patients, with both acute and chronic illnesses. In order to guarantee a prompt and specialized treatment of ED-attending cancer patients and reduce inappropriate inpatient admissions, a dedicated ED cancer pathway (EDCP) consisting of EDbound Medical Oncology (MO) resident doctor and direct admission for candidate patients exclusively to the MO division was established at the Tor Vergata University Hospital in April 2015. Patients and Methods: Consecutive cancer patients attending the ED in two reference three-month periods were enrolled: pre-EDCP period, from 1st October 2014 to 31st December 2014, and post-EDCP period, from 1st October 2014 to 31 st December 2015. Inpatient admission rate, mortality rate and both ED and inpatient length of stay were compared between the two analyzed periods, pre- and post-EDCP. Results: In the pre-and post-EDCP periods 127 and 123 cancer patients, respectively, were included. Most of the analyzed indicators were improved by EDCP implementation: Inpatient admission rate from $70 \%$ to $41 \%$ ( $p<0.0001)$, ED mortality rate from 10-4\% ( $p=0.04)$, mean ED length of stay, from 58 to $42 \mathrm{~h}$ $(p=0.03)$, mean inpatient length of stay, from 15.5 to 6.5 days $(p<0.0001)$, in the pre- and post-EDCp period, respectively. Conclusion: EDCP implementation led to a significant improvement of health care delivery to cancer patients attending the Emergency Department.
\end{abstract}

Emergency departments (EDs) often face overcrowding issues while simultaneously confronting with the increasing

Correspondence to: Jacopo Maria Legramante, Emergency Department, Tor Vergata University Hospital, Viale Oxford, 81, 00133, Rome, Italy. Tel: +39 0620908211, e-mail: legraman@uniroma2.it

Key Words: Cancer patients, emergency department, cancer patient hospitalization. clinical needs of patients, such as cancer patients, with both acute and chronic illnesses. Cancer is the second leading cause of death worldwide after cardiovascular disease, with an increasing number of elderly adults with cancer having complex health needs (1).

It is widely acknowledged that more active treatments and improved supportive care have significantly prolonged survival among patients with cancer.

During this prolonged and sometimes tortuous disease history, cancer patients, especially those with advanced disease, may present complications or significant clinical deterioration requiring emergency care as a result of 1) declining clinical history, 2) toxicity of administered anti-cancer therapies 3) inappropriate symptom management. Also, the near end-of-life period may be characterized by frequent accesses to the ED, and this may be indicative of inadequate health care in the community (e.g. home health or palliative care services).

In particular for near end-of-life patients, palliative management within the emergency-care setting might be suboptimal with consequent unnecessary suffering for these patients (2).

Population aging with increased cancer incidence and prevalence as well as broadened anticancer therapeutic armamentarium will probably lead to even more cancerrelated ED utilizations in the near future. ED overcrowding is therefore becoming a major challenge for ED physicians and it is a psychological 'nightmare' for the patients.

In this respect, it appears mandatory to minimize the stay of cancer patients in the emergency room and define specific management plans for immediate patient care and rapid definition of the overall clinical strategy regarding the inpatient admission $v s$. extra-hospital care.

In April 2015, for the purpose of prompt and specialized treatment of ED-attending cancer patients, an ED-specific cancer clinical pathway (EDCP) was established at the Tor Vergata University Hospital. EDCP was authorized and supervised by the Tor Vergata Hospital General Directorate 
and operated at different levels by: 1) establishing the continuous presence of in-training Medical Oncology (MO) resident doctors in the $\mathrm{ED}$, who will be closely linked with the MO specialist who cooperates with the emergency physicians in order to choose the best clinical strategy for cancer patients; 2) reserving a part of the Medical Oncology Division for the direct inpatient admission from Emergency Room; 3) setting up a dedicated pathway toward palliative care facilities for oncology patients not candidate for active therapies.

All inpatient admissions were authorized after discussion with the Medical Directorate of Tor Vergata University hospital.

In the present study, we analyzed the impact of EDCP since its establishment in terms of efficiency indicators in the management of cancer patients in both ED and MO Division.

\section{Patients and Methods}

The study included adults ( $>18$ years) patients with a solid tumor diagnosis according to ICD-9 coding system who attended the Emergency Room of Tor Vergata University Hospital.

Patients discharged at home within four hours were excluded from the analysis, since their ED visit was considered inappropriate by the ED physician.

Two samples of patients were arbitrarily chosen based on two distinct three-month periods of observation. The first group included cancer patients referred to ED from October 2014 to December 2014 when the EDCP (see below for details) was not active, and the second group included ED cancer patients from October 2015 to December 2015, after the activation of EDCP.

EDCP. The Emergency Department Cancer Pathway (EDCP) was established in June 2015 and aimed at strengthening the collaboration between the ED and the MO division by guaranteeing the presence in the Emergency Room during daytime $(12 \mathrm{~h})$ of at least one in-training MO resident doctor who could take over cancer patients attending the ED and plan the optimal clinical strategy in cooperation, via phone consultation, with the MO specialist in charge of the MO ward. The period of training of the MO resident doctor in the ED was formally included in the academic residency program.

Examples of decisions taken by means of EDCP were: patient disposition for inpatient admission $v s$. release at home $v s$. transfer to palliative care facilities vs. other type of oncology-oriented palliative treatment; in- and out-hospital diagnostic plans.

Palliative management outside the Tor Vergata University Hospital was ensured by an established collaboration between the Hospital and palliative care facilities as certified by the European Society of Medical Oncology (http://www.esmo.org/Patients/DesignatedCentres-of-Integrated-Oncology-and-Palliative-Care/OncologiaMedica-Policlinico-Universitario-Tor-Vergata-Rome-San-RaffaeleHospices-Rocca-di-Papa-Montecompatri-Italy).

The MO resident doctor also helped arranging subsequent procedures or investigations that patients would need once outside the ED. When indicated, EDCP included the specific arrangement for admission of cancer patients exclusively to the MO ward, while before EDCP cancer patients were admitted to the Internal Medicine ward.
Table I. Characteristics of 250 enrolled patients.

\begin{tabular}{lccc}
\hline Characteristics & $\begin{array}{c}\text { Total: } \\
250 \mathrm{pts}\end{array}$ & $\begin{array}{c}\text { Pre-EDCP: } \\
127 \mathrm{pts}\end{array}$ & $\begin{array}{c}\text { Post-EDCP: } \\
123 \mathrm{pts}\end{array}$ \\
\hline Gender & & & \\
Male & $61 \%$ & $57 \%$ & $68 \%$ \\
Female & $39 \%$ & $43 \%$ & $32 \%$ \\
Age & & & \\
Median & 73 years & 70 years & 74 years \\
Range & $(22-93)$ & $(22-85)$ & $(38-93)$ \\
Primary tumor & & & \\
Lung & $33 \%$ & $30 \%$ & $39 \%$ \\
Breast & $12 \%$ & $13 \%$ & $10 \%$ \\
GI tract & $33 \%$ & $35 \%$ & $29 \%$ \\
Prostate & $8 \%$ & $8 \%$ & $8 \%$ \\
Other & $14 \%$ & $14 \%$ & $14 \%$ \\
\hline
\end{tabular}

Pts: Patients; GI: gastrointestinal; EDCP: Emergency Department Cancer Pathway.

Efficiency measures. Four efficiency indicators were measured to evaluate the potential improvement in patient management with the introduction of EDCP: 1) proportion of ED cancer patients admitted as inpatient, 2) length of stay (measured in hours) in the emergency room before inpatient admission, 3) length of stay (measured in days) in hospital after admission as inpatient in the Internal Medicine Department in the pre-EDCP period $v s$. admission in the MO Division in the EDCP period, 4) mortality during the ED stay.

Statistical analyses. Length of stay (measured either in hours or days) was checked for normality distribution by the Kolmogorov-Smirnoff test. T test for independent samples with Welch test to correct for unequal variances was used to compare lengths of stay between the pre-EDCP period (year 2014) and post-EDCP period (year 2015). Differences in categorical variables (inpatient admission rate and mortality) were compared using the chi-square test. Values are expressed as means \pm SEM (standard error of the mean). Differences were considered statistically significant for $p$-values $<0.05$.

\section{Results}

A total of 250 consecutive cancer patients attending the ED in the selected periods were included in the study: 127 patients between October and December 2014 (pre-EDCP period) and 123 patients between October and December 2015 (post-EDCP period). ED visits of patients with a cancer diagnosis constituted nearly $5 \%$ of the over 5000 ED visits recorded in the analyzed time frames. Characteristics of the 250 enrolled patients are depicted in Table I.

As for the tumor subtype classification, most frequent tumor diagnoses were lung cancer (33\% of cases), cancer of the gastrointestinal tract $(33 \%)$ and breast cancer $(12 \%)$ (Table I).

In the pre-EDCP period, 89 out of 127 ED cancer patients, representing the $70.1 \%$ of patients, were admitted to the 


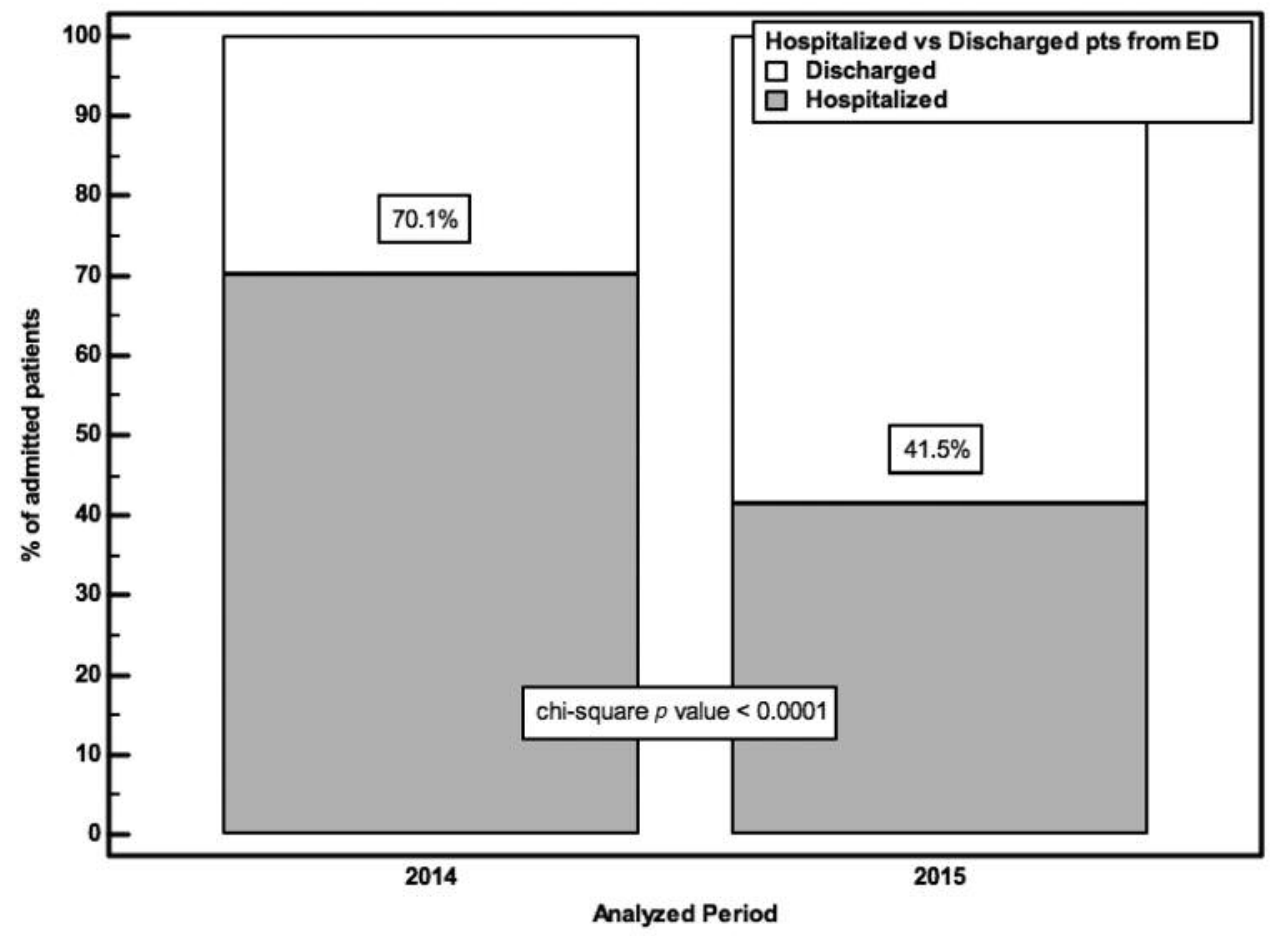

Figure 1. Inpatient admission rate of cancer patients attending the Emergency Department in the two analyzed time periods.

Internal Medicine Department, whereas in the post-EDCP period 51 out of $123 \mathrm{ED}$ cancer patients (41.5\% of patients) were admitted to the Medical Oncology division, with an overall statistically significant reduction (chi square $p<0.0001$ ) in inpatient admission rate (Figure 1). ED cancer patients not admitted to the Institution of Tor Vergata Hospital either were discharged at home, transferred to other tertiary hospitals, transferred to palliative care institutions or died in the ED.

In the 2014 period, when the EDCP was not active, 13 cancer patients $(10.2 \%$ of analyzed cancer patients) died in the emergency room, whereas in 2015, when EDCP was active, 6 cancer patients $(4.9 \%)$ died in the ED (chi square $p=0.04)$.

A significantly longer stay in the ED before disposition was observed for cancer patients in the pre-EDCP period, when patients were managed exclusively by the emergency physicians without the help of the MO resident doctor. Mean length of ED stay in the 2014 pre-EDCP $v s .2015$ post-EDCP period was $58.4 \mathrm{~h} v s .41 .9 \mathrm{~h}$, respectively, $p=0.0295$ (Figure 2).

For patients admitted to Tor Vergata divisions, the mean length of stay in the inpatient setting was significantly inferior in the 2015 period, when the MO-specific admission was active: 6.5 vs. 16.5 days for the post- $v s$. pre-EDCP period, respectively $p<0.0001$ (Figure 3).
Inpatient mortality after admission did not significantly differ between the 2014 period and the 2015 period, with 6 out of 89 admitted cancer patients dying in the Internal Medicine division (6\%) vs. 1 out of $51(2 \%)$ in the Medical Oncology division (chi square $p=0.397$ ).

\section{Discussion}

In the present pilot study, we confirmed the significant improvement of health care delivery when a dedicated specialty track is set up in the ED. The burden of cancerrelated ED visits (5\% of all ED visits) and the inpatient admission rate of ED cancer patients (70\% in 2014 and $41 \%$ in 2015) were comparable to those reported in previous studies (3). In an analysis of nationwide data in US, the proportion of adult cancer-related ED visits were reported to be $4.2 \%$, with an inpatient admission rate of $59.7 \%$ for cancer patients as compared to $16.3 \%$ for non-cancer-related ED patients (3).

Cancer is currently the second leading cause of death worldwide (4). Population aging, especially in western countries, has been recognized as a major determinant in the increase of cancer incidence in the last decades. Aging comes along with degenerative comorbidities and evidence exists that comorbidity indexes are independent predictors of ED attendance by cancer patients (5). Furthermore, 


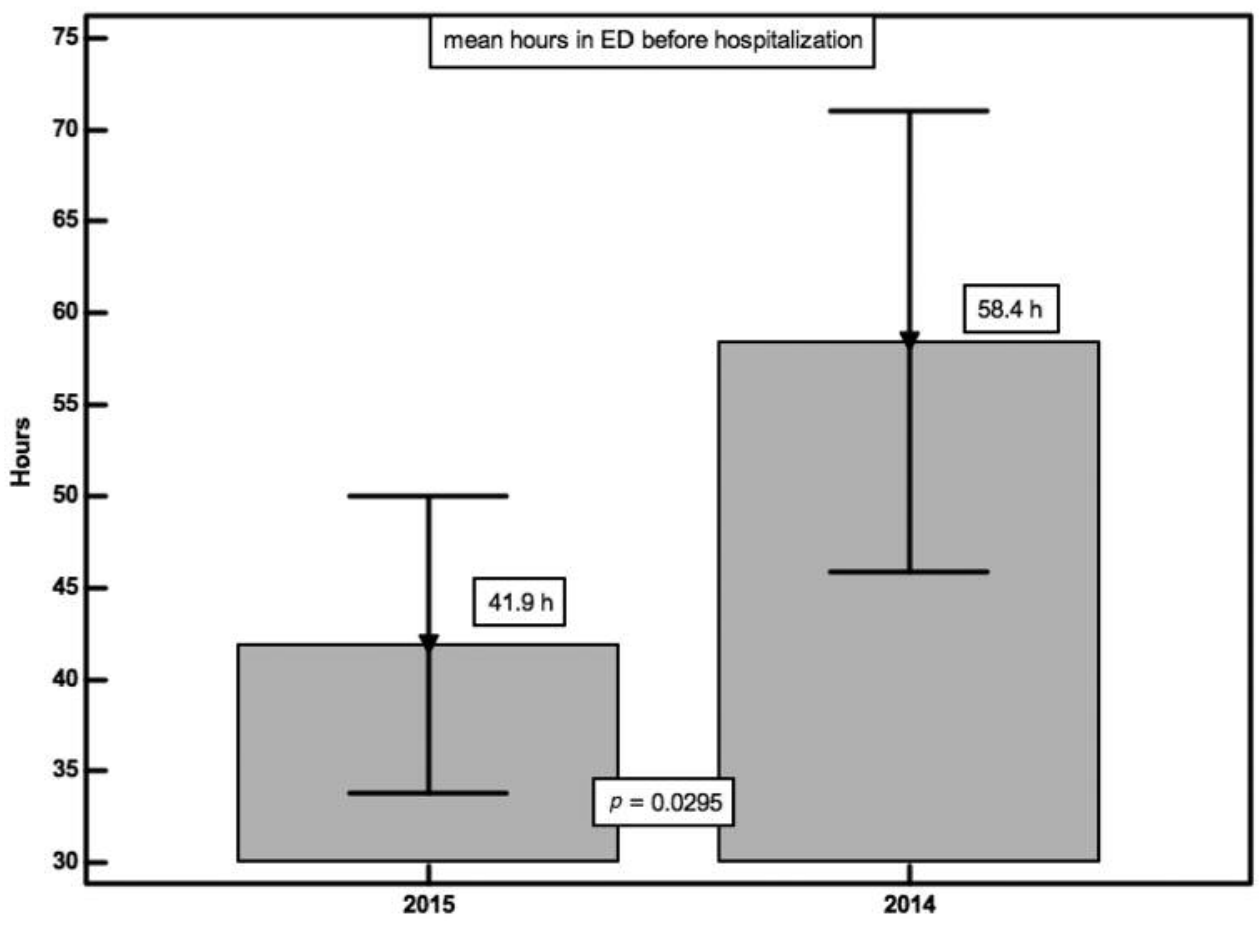

Figure 2. Length of stay of cancer patients in the Emergency Department before disposition in the two analyzed time periods.

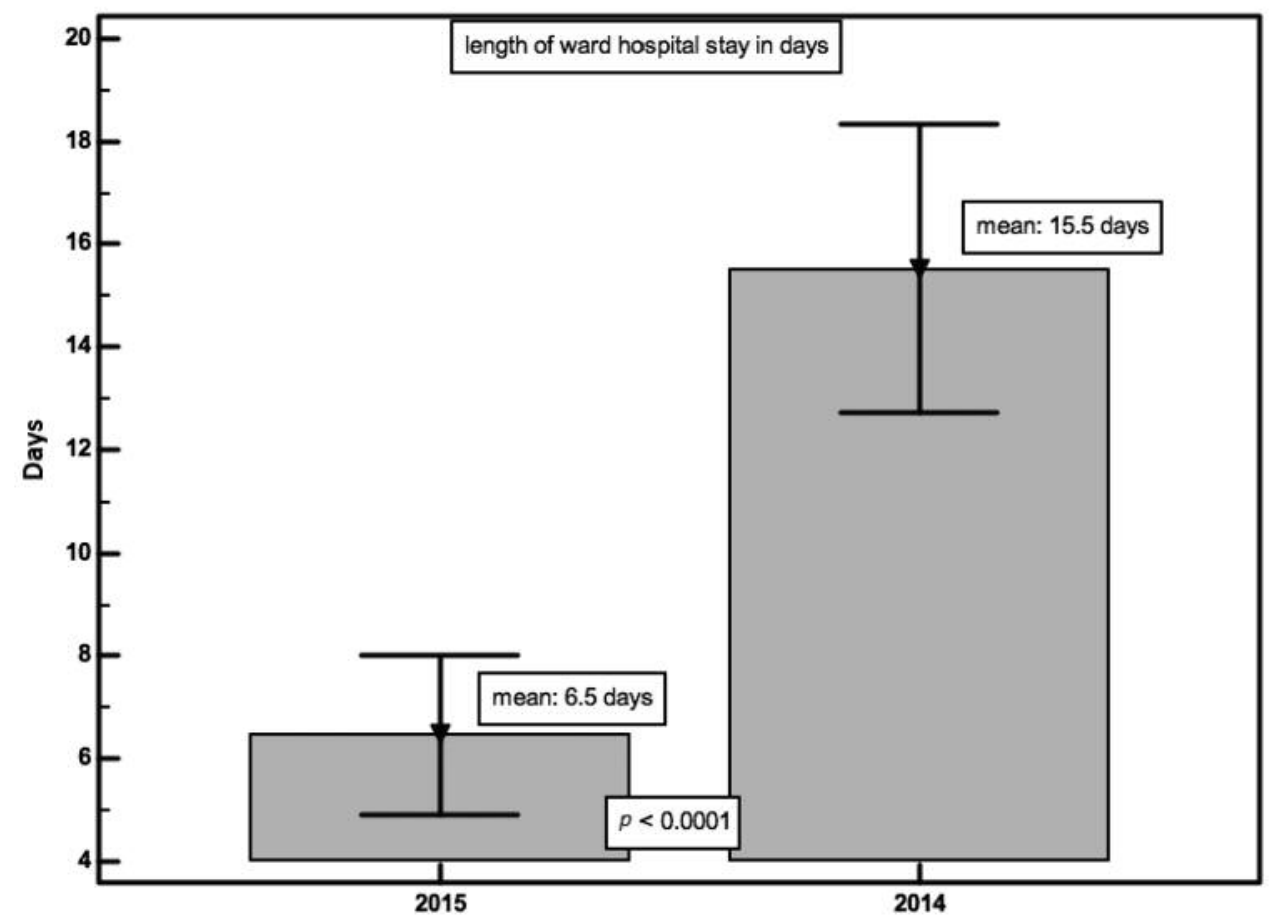

Figure 3. Length of inpatient stay of cancer patients after admission from the Emergency Department in the two analyzed time periods. 
advancements in diagnostic equipments, cancer treatments and supportive care over the past two decades have resulted in a significant improvement in survival rates. Accordingly, cancer patients have been facing longer disease durations frequently characterized by treatment and disease-related acute complications requiring ED utilization (6).

Establishment of early and proper treatment of these patients in the ED might substantially enhance their quality of life and decrease mortality rate (7).

In an attempt to optimize the management of cancer patients in the emergency room, in the spring of 2015 we set up a dedicated route managed by the emergency physician with the relevant contribution of a Medical Oncology resident doctor in collaboration with the Specialist Medical Oncologist in charge of the inpatient setting.

To evaluate the impact of this new route we monitored different parameters that might express the quality of delivered health care. Amongst them were 1) the rate of cancer inpatient admission, 2) the length of stay both in the ED and in the inpatient setting 3 ) the mortality rate both in the ED and during admission.

Data clearly showed that a preferential route for cancer patients produced a substantial and statistically significant improvement in almost all analyzed quality parameters.

In particular, a significant reduction in inpatient admission rate was observed from 2014 to 2015 which is likely due to the proper management of cancer patients with avoidance of unnecessary hospitalizations. Furthermore, the specialistoriented treatment of these patients has to be considered the most significant contributor of decreased mortality observed in our study (8).

A further optimization in the quality of care achieved by EDCP was the significant reduction in the length of both ED and inpatient stay (Figures 2 and 3).

Various favorable secondary effects were also associated to EDCP. Firstly, a significant reduction in the costs of hospital care. In fact, the rise of health care-related expenditures is mainly driven by two factors: hospital-related expenditures and drug prices. Secondly, the reduction in the length of stay in the ED minimized ED overcrowding. Finally, restraining prolonged hospital stays had a clear beneficial effect on patient's psychological health and reduced the risk of hospital-acquired infections.

One of the achievements of EDCP was the early activation of palliative care management.

Early supportive care of cancer patients has clearly demonstrated to prolong survival, improve health-related quality of life and reduce unnecessary hospitalization and ICU admission (9).

There are some important limitations of the present study, above all the monocentric nature of the data source which may limit the external validity of our findings. However, to the best of our knowledge, this is the first report on a dedicated cancer pathway active in an Emergency Department and its extension as a multi-centric cooperation is warranted.

In conclusion, our data clearly showed that a specific and dedicated pathway for cancer patients active in the ED is effective in reducing the inappropriate hospitalization of these patients and that, when hospitalization is required, the length of stay both in the emergency room and in the hospital's divisions is significantly reduced. Even more importantly, EDCP is associated with a significant and substantial reduction in mortality rate during the ED stay.

\section{References}

1 Swenson KK, Rose MA, Ritz L, Murray CL and Adlis SA: Recognition and evaluation of oncology-related symptoms in the emergency department. Ann Emerg Med 26: 12-17, 1995.

2 Royal College of Physicians and Royal College of Radiologists. Cancer patients in crisis: responding to urgent needs. Report of a working party. London: RCP, 2012. [cited 2013 Dec 12]. Available from: http://www.rcplondon.ac.uk/sites/ default/files/ documents/cancer-patients-in-crisis-report.pdf

3 Rivera DR, Gallicchio L, Brown J, Liu B, Kyriacou DN and Shelburne N: Trends in adult cancer-related emergency department utilization: An analysis of data from the nationwide emergency department sample. JAMA Oncol 3: e172450, 2017.

4 Torre LA, Bray F, Siegel RL, Ferlay J, Lortet-Tieulent J and Jemal A: Global cancer statistics, 2012. CA Cancer J Clin 65: 87-108, 2015.

5 Uramoto H, Iwashige A, Kagami S and Tsukada J: Prediction of emergency hospitalization of outpatients receiving cancer chemotherapy. Anticancer Res 27: 1133-1136, 2007.

6 Temel JS, McCannon J, Greer JA, Jackson VA, Ostler P, Pirl WF, Lynch TJ and Billings JA: Aggressiveness of care in a prospective cohort of patients with advanced NSCLC. Cancer 113: 826-33, 2008.

7 Mayer DK, Travers D, Wyss A, Leak A and Waller A: Why do patients with cancer visit emergency departments? Results of a 2008 population study in North Carolina. J Clin Oncol 29: 26832688,2011

8 Tanriverdi O, Beydilli H, Yildirim B and Karagoz U: Single center experience on causes of cancer patients visiting the emergency department in southwest Turkey. Asian Pac J Cancer Prev 15: 687-690, 2014.

9 Temel JS, Greer JA, Muzikansky A, Gallagher ER, Admane S, Jackson VA, Dahlin CM, Blinderman CD, Jacobsen J, Pirl WF, Billings JA and Lynch TJ: Early palliative care for patients with metastatic non-small-cell lung cancer. N Engl J Med 363: 73342, 2010. 